\title{
Editorial \\ Special issue on single chain polymers
}

Nanoscience and nanotechnology have been driven, to a large extent, by key enabling approaches and platforms, including characterization techniques such as atomic force microscopy, optical tweezers, force clamps, single molecule fluorescence, laser scanning confocal microscopy, and near field optics. These new techniques allow one to image and manipulate single polymers, as well as to study their dynamics at the single molecule level, hence promoting significantly the progress of traditional polymer science and opening many new horizons for the future.

In view of the swift progress and great impact of current research aiming at single chain polymers, we have edited this special issue in an attempt to bring together, under a single cover, representative examples encompassing achievements in chemistry, physics, and life sciences. The special issue includes 13 papers from eight countries. The topics cover many areas at the current frontiers of science of single chain synthetic polymers, as well as biomacromolecules, including redoxdriven single chain motors, interfacial conformation of polymers, single chain elasticity, force induced conformational transitions, single molecule imaging, host-guest intermolecular interactions, folding and unfolding dynamics of biomacromolecules, macromolecular crowding effects, and mechanochemical switches. Although the choice of subjects included in this special issue is not intended to be comprehensive, it can give the reader an overview of the state-of-the-art of this rapidly growing field. It is our hope that the special issue will serve as a point of departure to continue creative studies in this multidisciplinary research area.

\section{Acknowledgements}

It is our great pleasure to acknowledge all the invited authors for the submission of their excellent work. We also thank James E. Mark, Editor of Polymer, for his initiative to launch this section, and for his assistance with editing this special issue.

$$
\begin{array}{r}
\text { Xi Zhang* } \\
\text { Key Lab of Organic Optoelectronics and Molecular } \\
\text { Engineering, Department of Chemistry, } \\
\text { Tsinghua University, Beijing 100084, } \\
\text { People's Republic of China } \\
\text { E-mail address: xi@ mail.tsinghua.edu.cn } \\
\text { G. Julius Vancso } \\
\text { MESA + Institute for Nanotechnology, } \\
\text { University of Twente, 7500 AE Enschede, } \\
\text { The Netherlands } \\
\text { E-mail address: g.j.vancso@ @nw.utwente.nl }
\end{array}
$$

Available online 7 February 2006 